\title{
STEAM, ¿una nueva distracción para la enseñanza de la ciencia?
}

\author{
Antonio García-Carmona \\ Departamento de Didáctica de las Ciencias Experimentales y Sociales. \\ Universidad de Sevilla. España
}

[Recibido el 24 de mayo de 2020, aceptado el 24 de agosto de 2020]

Se presenta un ensayo sobre la irrupción del movimiento STEAM (science, technology, engineering, arts and mathematics) en el contexto educativo español. El ensayo, de naturaleza crítica y reflexiva, se construye desde la mirada particular, pero informada, de un educador de profesorado de ciencia, que analiza la aportación, pertinencia y viabilidad del movimiento STEAM en el complejo proceso de mejora que requiere la educación científica básica en España. Para ello, se empieza con una reflexión en torno al significado del acrónimo inglés y su relación con el tradicionalmente denominado ámbito curricular científico-tecnológico. Luego se discute sobre la perspectiva de STEAM como posible enfoque educativo, la analogía escolar del STEAM profesional y las competencias STEAM. A continuación, se analiza el encuadre del movimiento STEAM con la concepción tradicional de la enseñanza de la ciencia. Se termina con una reflexión global acerca de las perspectivas que pueden augurarse respecto a STEAM como enfoque educativo en el contexto español actual.

Palabras clave: ámbito científico-tecnológico; contexto educativo; enfoque educativo; enseñanza de la ciencia; STEAM

\section{STEAM-A new distraction for science education?}

An essay about the irruption of STEAM (science, technology, engineering, arts and mathematics) movement in the Spanish educational context is presented. The essay, which is critical and reflective in nature, is built from the particular view, yet informed, of a science teacher educator, who analyses the contribution, pertinence and viability of the STEAM movement in the complex process of improvement that basic science education requires in Spain. To this end, firstly a reflection around the meaning of the English acronym and its relationship with the traditionally known as curricular area of science and technology is made. Secondly, it is discussed about STEAM as a possible educational approach, the school analogy of professional STEAM, and the STEAM competences. Thirdly, the fit of STEAM with the traditional conception of science education is analysed. Finally, a global reflection about what perspectives regarding STEAM as an educational approach can be expected in the current Spanish context is exposed.

Keywords: educational approach; educational context; science education; scientific-technological area; STEAM

Para citar el artículo. García-Carmona, A. (2020). STEAM, ¿ una nueva distracción para la enseñanza de la ciencia? Ápice. Revista de Educación Científica, 4 (2), 35-50. DOI: https://doi.org/10.17979/ arec.2020.4.2.6533

Contacto.garcia-carmona@us.es 
Lo invisible solo existe porque no se ve. Fito \& Fitipaldis

\section{STEAM, el término inglés que todo lo impregna}

Es difícil negar que, en los últimos años, todo lo relacionado con el movimiento STEM (science, technology, engineering and mathematics) ${ }^{1}$, o STEAM si se añade la A de 'arts', tiene un protagonismo especial en el ámbito de la didáctica de la ciencia ${ }^{3}$. Se trata, sin ninguna duda, del término de moda, y mencionarlo en publicaciones, proyectos, congresos, cursos, redes sociales, etc., parece situarte a la vanguardia de lo que se está innovando en educación científico-tecnológica. Desde mi punto de vista, el mensaje que emana de ello es meridianamente claro, a la vez que pernicioso: "Ponga STEAM en su vida académica, y todo le irá mejor". O, parafraseando el título de una canción de Amaral, "Sin STEAM no soy nada".

STEAM se fomenta, además, de manera incesante mientras buena parte del profesorado no tiene claro qué implica una educación enmarcada en este movimiento (Pastor, 2018; Radloff y Guzey, 2016), ni reúne, en su inmensa mayoría, las competencias docentes necesarias para llevarla a cabo en el aula con garantías y autonomía suficientes (Ejiwale, 2013; Margot y Kettler, 2019; Toma y Greca, 2018). Esta dificultad se acentúa de manera significativa cuando STEAM se intenta promover en los niveles educativos más básicos. La situación me recuerda al personaje de la comedia de Calderón de la Barca, "El niño bien criado", quien, para reclamar su porción de comida al padre olvidadizo, dice en voz alta al gato que maullaba a su alrededor: "¿de qué me pides los huesos, si aún no me han dado la carne?"

En España, a diferencia de otros enfoques y constructos didácticos también importados del ámbito anglosajón, STEAM se ha acuñado directamente con el acrónimo en inglés. En español sería CTIAM, pero su traducción no parece haber cuajado. ${ }^{4}$ Así que, no queda claro si debemos pronunciar STEAM en inglés ("estim"), o tal y como suena en nuestro idioma ("esteam"). Porque, hoy por hoy, no es un anglicismo, según la Real Academia Española de la Lengua. En consecuencia, me adhiero a las reflexiones de Acevedo-Díaz (2020) y Martín-Gordillo (2020) acerca de las posibles connotaciones perversas que pueden derivarse de usar el término inglés STEAM. Efectivamente, entre sus significados están los sustantivos "vapor" e "ímpetu", así como el verbo "echar vapor", que -caprichos del lenguaje- pueden invitar a pensar que el movimiento STEAM, tal vez, ha llegado al contexto educativo español para "vender humo" de forma un tanto "vigorosa".

Es obvio que la traducción de STEAM al español no cambia la sustancia que envuelve a este movimiento. Sin embargo, pienso que no se debería restar importancia al lenguaje e idioma manejados en los procesos de adopción de nuevas ideas o constructos en contex-

1 El movimiento STEM surge en Estados Unidos, durante la década de 1990, con un claro propósito económico-productivo: promocionar las disciplinas que constituyen el acrónimo para mejorar la competitividad del país (Toma y García-Carmona, 2019-en prensa).

2 Por economía del lenguaje, a lo largo del texto me referiré siempre a STEAM.

3 Lo escribiré así a lo largo de todo el texto, como una simplificación de didáctica de las ciencias experimentales.

4 En revistas españolas de investigación en didáctica de la ciencia, los dos únicos trabajos que he encontrado usando el acrónimo en español (CTIM, en este caso) son los de Rossi y Barajas (2015) y Vázquez y Manassero (2015), referidos a la cuestión de género en la elección de estudios relacionados con el acrónimo y a las vocaciones científico-tecnológicas en la educación superior, respectivamente. 
tos distintos de los que estos proceden; sobre todo, cuando llevan inherente una manifiesta carga ideológica. Conviene tener presente que el movimiento STEAM toma impulso en Estados Unidos con un claro trasfondo político-económico de corte neoliberal (Sharma, 2016; Stevenson, 2015; Takeichi, Sengupta, Shanahan, Adams y Hachem, 2020), ante el creciente y competitivo desarrollo científico-tecnológico de potencias mundiales emergentes como China (Perales y Aguilera, 2020). Además, esta perspectiva neoliberal de la educación STEAM se ha extendido a otros países, principalmente de cultura occidental, como reflejo de la Globalización hegemónica existente en el mundo actual (Bencze et al., 2019; Carter, 2017).

De forma paralela, también en Estados Unidos hay quienes ven en las reformas educativas asociadas a STEAM una nueva oportunidad para intentar mitigar las grandes desigualdades estructurales existentes en ese país, debidas principalmente a la cuestión racial (Vakil y Ayers, 2019). Aunque la experiencia con ello en algunas ciudades como Chicago indican que el reto se torna muy difícil (Morales-Doyle y Gutstein, 2019). En este mismo plano del movimiento STEAM, Chen y Buell (2018) llegan a hablar, incluso, de "proyecto racial neoliberal", al analizar el papel de la población asiática americana en el sistema STEAM estadounidense.

\section{STEAM, ¿una nueva denominación del "ámbito curricular científico-tecnológico"?}

Quizás lo de mantener el acrónimo STEAM en inglés sea para vestir con algo más de "glamur" al comúnmente denominado "ámbito curricular científico-tecnológico"; el cual, en nuestro contexto educativo siempre ha incluido las matemáticas, las ciencias de la naturaleza y la tecnología. Igualmente ha estado integrada la ingeniería que, de acuerdo con Raynaud (2018), es la parte de la tecnología dedicada a la producción de artefactos. Basta con revisar la historia de la educación en España para comprobar que esto último sucede así desde hace, al menos, medio siglo. En efecto, la Ley General de Educación (1970) ya establecía para la etapa educativa básica (más conocida como EGB) una materia escolar denominada "pretecnología", que promovía la construcción de sencillos artefactos. Luego, a partir de la entrada en vigor de la LOGSE (1990), y en las leyes educativas que se han ido sucediendo hasta el presente, existe el área de tecnología que promulga, de igual forma, el análisis, diseño y construcción de artefactos entre sus objetivos básicos.

No obstante, es preciso señalar que, a diferencia de la conexión curricular entre la ciencia y las matemáticas, que es obvia e ineludible desde un punto de vista epistemológico (García-Carmona, 2001), la integración educativa entre la ciencia y la tecnología ha sido históricamente más compleja de concebir (Maiztegui et al., 2002). Posiblemente porque la naturaleza de la tecnología (Acevedo-Díaz, 1998; Raynaud, 2018) no termina de ser bien comprendida. Y, desafortunadamente, ello se sigue percibiendo en las propuestas educativas bajo el prisma de STEAM (Pleasants, Clough, Olson y Millers, 2019).

Desde mi particular visión, uno de los principales obstáculos para una integración curricular adecuada entre la ciencia y la tecnología, que en su grado de máxima hibridación es la "tecnociencia" (Echeverría, 2005), se encuentra en identificar ingenuamente la tecnología solo con herramientas TIC. ${ }^{5}$ Sin restar, en absoluto, importancia a estas últimas en la

5 Lamentablemente, desde el ámbito de la pedagogía se está contribuyendo a esta concepción ingenua de la tecnología. Con suma frecuencia se habla de "tecnología en educación" para hacer referencia exclusiva al uso de herramientas TIC en los procesos de enseñanza/aprendizaje. Y esta misma perspectiva de la tecnología es la que suele acuñarse en las propuestas educativas STEAM (e.g., Dogan y Robin, 2015). 
enseñanza/aprendizaje de cualquier materia, tal perspectiva de la tecnología supone una degradación significativa de su estatus epistemológico. No en vano, la tecnología constituye un ámbito del saber humano poliédrico y multifacético, que está integrado por conocimientos teóricos, métodos de razonamiento y procesuales, técnicas, dispositivos, sistemas, organizaciones, etc. (Acevedo-Díaz, 1998; Acevedo-Díaz y García-Carmona, 2016; Criado y García-Carmona, 2011; Raynaud, 2018).

Con relación a la componente artística de STEAM, que suele referirse a la estética y creatividad dentro de las disciplinas que conforman el acrónimo, ¿̇acaso estos dos rasgos no son inherentes, por naturaleza, a las disciplinas científico-tecnológicas? Si se repasa la historia de la ciencia y la tecnología, se encuentra que los criterios estéticos y creativos han tenido un protagonismo especial de manera permanente. Como Aitken y Mills (1997) apuntan, la tecnología es una mezcla de ingenio, pericia e ingeniería creativa que surge cuando se busca satisfacer una necesidad humana o resolver un problema. Asimismo, Garratt (1993) señala con respecto al diseño tecnológico que, "aunque es esencial que un diseño funcione correctamente, ya que tenemos sentimientos y emociones, y estamos rodeados de las cosas que fabricamos, también es importante que "sea bonito»." (p. 25).

Algo similar puede decirse de la ciencia básica. El ilustre ingeniero, matemático y físico británico Paul Dirac (1902-1984) llegó a decir que la belleza de las leyes y teorías científicas es, cuando menos, tan importante como la cuestión de que estas cuadren con los experimentos (Sánchez-Ron, 2016); un aspecto del que también habla el Nobel de Física Steven Weinberg en su libro "El sueño de una teoría final" (Weinberg, 2010). Este autor afirma que "algo tan personal y subjetivo como nuestro sentido de la belleza nos ayuda no solo a inventar teorías físicas, sino incluso a juzgar la validez de las teorías" (p. 277). Luego, añade:

La belleza que encontramos en teorías físicas como la relatividad general o el modelo estándar es muy parecida a la belleza que les confiere a algunas obras de arte la sensación de inevitabilidad que nos sugieren: la sensación de que uno no quisiera cambiar una sola nota o una pincelada o una línea. (Weinberg, 2010, p. 281)

\section{STEAM como enfoque educativo}

Lo primero que recuerdo haber leído sobre STEAM en español (no quiero decir que se trate de lo primero publicado en nuestro idioma), fue el artículo de Ocaña, Romero, Gil y Codina (2015); un equipo de docentes del área de tecnología que promovió la implantación de asignaturas de robótica en la ESO y Bachillerato en institutos de la provincia de Almería. He de reconocer que, desde aquel momento, y durante cierto tiempo, me limité a asociar STEAM con robótica escolar. Luego, cuando llegó la gran avalancha promocional del movimiento STEAM, comprendí que con este se intenta ir más allá educativamente hablando.

El asunto más controvertido, en mi opinión, es que STEAM no se presenta solo como una designación del conjunto de personas y/o de asuntos relacionados con la ciencia, la tecnología, la ingeniería y las matemáticas, incluyendo la perspectiva artística. Si fuese así, creo que habría muy poco que discutir. La cuestión es que STEAM se promueve, también, como un "enfoque educativo" que propugna la integración sinérgica de varias de las materias o áreas curriculares que componen el acrónimo (Dare, Ellis y Roehrig, 2018; Kelley y Knowles, 2016; Takeichi et al., 2020); y eso ya es algo que me parece mucho más serio. Para algunos autores (e.g., Sanders, 2009), una educación STEAM se estaría dando si hay una integración de, al menos, dos áreas del acrónimo. Pero si la exigencia es tan liviana, 
teniendo en cuenta que STEAM representa a un total de cinco disciplinas, ciertamente considero que "para este viaje no se necesitan alforjas", porque ese planteamiento -con distintas acepciones y propuestas- ya existe, desde hace años, en el ámbito de la educación científico-tecnológica (Toma y García-Carmona, 2019-en prensa).

STEAM aparece, asimismo, como un nuevo enfoque educativo de integración curricular, después de que propuestas anteriores, enmarcadas en la idea de fusionar varias materias escolares del ámbito científico-tecnológico, hayan resultado poco eficaces y de escasa trascendencia en la práctica educativa (Czerniak, Weber y Ahern, 1999; Gil, 1994; Guerra, 1984; Maiztegui et al., 2002; McComas y Burgin, 2020). ¿'Por qué con STEAM puede ser diferente, si la carrera docente y los planes de formación del profesorado concernido siguen prácticamente igual tras décadas (Montero y García-Carmona, 2018)?

A ello hay que sumar que el movimiento STEAM irrumpe en nuestro contexto educativo cuando todavía no han terminado de madurar otros enfoques y planteamientos didácticos, que también fueron sugeridos antes como apropiados. Me refiero, por ejemplo, al aprendizaje por proyectos, el aprendizaje basado en problemas, el aprendizaje mediante indagación, y el enfoque Ciencia-Tecnología-Sociedad (CTS), con sus distintas variantes y misceláneas. A tenor de la escasa investigación didáctica que suele hacerse dentro del aula (Manchón-Gordón y García-Carmona, 2018), en comparación con otros tipos de estudios, no se puede decir que tengamos evidencias robustas de cómo funcionan dichos planteamientos en el quehacer docente diario. Solo tenemos evidencias suficientes de que sigue imperando el modelo de enseñanza tradicional para las áreas curriculares del ámbito científico-tecnológico (Cañal, Criado, García-Carmona y Muñoz, 2013; Porlán, 2018).

Entonces, la pregunta es: ¿qué pruebas empíricas provee la investigación didáctica sobre la efectividad del enfoque STEAM en sí, y en comparación con otros enfoques educativos, para que nos planteemos acogerlo con optimismo y confianza en la realidad de nuestro contexto educativo? Pienso que solo cuando se disponga de pruebas favorables en tal sentido, se darán las condiciones académicas y profesionales apropiadas para ello (GarcíaCarmona, 2020). Porque, una cosa es hacer propuestas teóricas bien fundamentadas que, indudablemente, son necesarias para el avance de cualquier campo de investigación $-y$ en esto a la educación STEAM le queda mucho camino que recorrer, según algunas revisiones recientes de la bibliografía (e.g., Martín-Páez, Aguilera, Perales-Palacios y VílchezGonzález, 2019; Toma y García-Carmona, 2019-en prensa)-; y otra bien diferente es que esos planteamientos teóricos: (1) se transformen en propuestas didácticas concretas y factibles para una implementación genuina del enfoque, y (2) se sometan a evaluación rigurosa en las aulas para determinar cuáles son sus garantías pedagógicas.

\section{¿Científicos o "STEAMíficos"?}

El tsunami dialéctico en torno a STEAM es tal, que he llegado a preguntarme si a célebres personajes de la historia de la ciencia habría que dejar de llamarlos científicos para denominarlos, a partir de ahora, "STEAMíficos". Porque, a sus grandes dotes para pensar sobre los mecanismos que gobiernan los fenómenos de la naturaleza (esto es, una extraordinaria "capacidad de pensamiento y quehacer científico"), habría que añadir sus excepcionales "habilidades matemáticas" para parametrizar fenómenos y establecer leyes (e.g., Newton, Maxwell, Boltzman, Planck, Noether...), así como "destrezas tecnológico-ingenieriles" verdaderamente portentosas para el diseño de ingeniosos artefactos con los que llevar a cabo sus experimentos (e.g., Galileo, Lavoisier, Boyle, Hertz, Curie...). Y todo ello, por no mencionar la trascendencia de sus contribuciones, de manera directa o como fuentes 
de inspiración, en los campos del arte ${ }^{6}$ las humanidades (e.g., Descartes, Pascal, Leibniz, Einstein...). Algo que, por cierto, el movimiento STEAM ha tratado de atender también, al menos parcialmente. Digo en parte porque, si bien intenta integrar explícitamente el arte, la educación STEAM olvida o no enfoca adecuadamente la perspectiva social y humanística ligada al desarrollo científico-tecnológico (Martín-Gordillo, 2020; Ortiz-Revilla, Greca y Adúriz-Bravo, 2020; Pleasants, 2020). Una perspectiva que sí es atendida de manera clara en otros enfoques integradores como, por ejemplo, la educación CTS (Aikenhead, 2005; Vázquez-Alonso, Acevedo-Díaz y Manassero-Mas, 2005).

Al hilo de todo esto, quisiera comentar también que encuentro cierta falacia en el discurso del movimiento educativo STEAM cuando se apela a que, con este, se promueve una analogía escolar del quehacer de los profesionales de esas materias, como si estos fuesen profundos conocedores del "totus integrated". Que profesionales de la ciencia, ingeniería, matemáticas, etc. trabajen juntos en proyectos inter o metadisciplinares, no significa necesariamente que sean especialistas en las distintas disciplinas que entran en juego; o, en otras palabras, que tengan un conocimiento holístico de todas las áreas implicadas en un proyecto STEAM. Entre otras razones, porque la formación académica de estos profesionales está estructurada en una amplia variedad de especialidades. Solo hay que ver la gran cantidad de títulos diferentes de grado y postgrado en las ramas de ciencias e ingenierías, que las universidades ofertan cada año. Esta política educativa tiene su origen en la Revolución Industrial (s. XVIII), que dio lugar a un mundo laboral basado en el trabajo especializado y en cadena. Consecuentemente, el ámbito de la educación se ha venido adaptando, de forma progresiva, a esta realidad con programas de cualificación profesional cada vez más desgranados en "microespecialidades".

Así pues, lo más habitual en un proyecto catalogable de STEAM es que cada profesional contribuya al desarrollo de este desde su parcela de conocimiento. Buena prueba de ello es lo que ha sucedido, por ejemplo, en la reciente investigación que ha logrado la primera imagen de un agujero negro. Según la información que trascendió a los medios sobre la participación española en este proyecto (Valdeolmillos, 2019), sabemos que el astrónomo del Instituto Astronómico de Andalucía (CSIC), Luis Gómez, se encargó de desarrollar uno de los tres algoritmos utilizados en la toma de la fotografía; que el también astrónomo Iván Martí-Vidal, del Instituto Geográfico Nacional, se ocupó de estudiar los campos electromagnéticos alrededor del agujero. Mientras que un grupo de científicos e ingenieros del radiotelescopio IRAM, situado en Sierra Nevada (Granada), trabajó en la configuración del equipo técnico para capturar la imagen.

\section{STEAM y el desarrollo de la competencia científico-tecnológica}

Si el trabajo real en STEAM suele darse en las condiciones antes comentadas, ¿tiene sentido o es educativamente viable esperar que, con el enfoque STEAM, el alumnado desarrolle una "competencia holística" relacionada con el conjunto integrado de áreas que conforman el acrónimo? El asunto se antoja de suma complejidad, por no hablar de desafío utópico, dentro de la realidad educativa actual. Aunque también es lícito, y en cierta medida necesario, creer en ese ideal educativo. Pero si lo que persigue la educación STEAM, en el fondo, es mejorar la competencia científico-tecnológica de la ciudadanía, no viene a aportar nada nuevo. Desde hace años, la enseñanza de la ciencia "per se" apunta entre sus finalidades el desarrollo de una alfabetización científica básica, que incluye el

6 Según Katona (2017), parece que fue notoria la influencia de la Teoría de la Relatividad, de Albert Einstein, en el pintor Salvador Dalí, cuando crea su famoso cuadro de "Los relojes blandos"; o en el poeta Federico García Lorca, al escribir su breve poema "Meditación primera y última". 
empleo de herramientas TIC y otros recursos, así como habilidades matemáticas. Todo ello, además, contextualizado en los problemas socioambientales, políticos, económicos, culturales, etc., que nos atañen como individuos y como sociedad (Cetto, Schneegans y Moore, 2000; OECD, 2006, 2019).

Asimismo, desde un punto de vista pragmático y factible, podría entender que la educación STEAM no pretendiera integrar también aspectos de las ciencias sociales y humanidades, ${ }^{7}$ de acuerdo con lo señalado antes. Si no, sería una empresa educativa tan pretenciosa que me lleva a pensar en la "consiliencia". Este término fue adoptado, hace algo más de dos décadas, por el entomólogo Edward O. Wilson para referirse a la "unidad de todo el conocimiento" (Wilson, 1999). Y el adjetivo más apropiado para hacer alusión a una persona con cualidades semejantes a las de esos personajes insignes del campo de la ciencia, aludidos más arriba, ya está inventado: "ilustrada". Una denominación que cobró un valor trascendente durante el siglo XVIII con el movimiento filosófico, político y cultural que promulgaba la razón y el conocimiento como bases del progreso social; es decir, la concepción de una sociedad orientada por la ciencia (García-Carmona, 2002).

\section{STEAM y el rigor científico de una disciplina académica}

Para mí, lo preocupante del movimiento educativo STEAM ya no es solamente (Toma y García-Carmona, 2019-en prensa):

(i) que no haya sido conceptualizado adecuadamente para hacerlo factible y operativo en las aulas, sin caer en meras "caricaturas" de lo que sería un enfoque STEAM integrado auténtico;

(ii) que una parte importante de propuestas educativas enmarcadas bajo el prisma de STEAM sean, en realidad, aquellas que hasta hace relativamente poco eran propuestas de enseñanza de la ciencia;

(iii) que el enfoque STEAM integrado no haya superado aún unas pruebas mínimas de validez educativa exportables a contextos diversos; $y$

(iv) que no exista un marco bien fundamentado y específico de "conocimiento didáctico del contenido" para la educación STEAM, ni sobre la naturaleza epistemológica de ese conglomerado de materias ${ }^{9}$, con vistas a diseñar: (1) programas de formación de profesorado y (2) propuestas de enseñanza realistas para su implementación en las aulas.

7 Obviamente, esto no se debería confundir con la necesidad educativa de contextualizar los contenidos escolares del ámbito científico-tecnológico en la realidad social y cultural que circunda al alumnado.

8 Es decir, no una mera yuxtaposición de algunas de las áreas o disciplinas que componen el acrónimo STEAM, sino una integración "transdisciplinar" de estas, con todo el "corpus" didáctico, epistemológico y axiológico que ello requeriría.

9 Sin ánimo de detenerme apenas en esto, creo necesario añadir que esta preocupación ha sido manifestada también por otros autores (Akerson et al., 2018; Ortiz-Revilla et al., 2020; Reynante, Selbach-Allen y Pimentel, 2020), porque solo conocemos, si acaso, la naturaleza epistemológica de algunas de las áreas de conocimiento que componen STEAM (Toma y García-Carmona, 2019-en prensa). Sin embargo, el carácter transdisciplinar del enfoque STEAM integrado lo proyecta como un constructo con entidad propia, que va más allá de lo que constituye cada una de esas áreas por separado. Con lo cual, se hace imprescindible establecer cuáles son los rasgos epistemológicos de STEAM como un "todo", en aras de hacer transposiciones didácticas apropiadas de este. Pero esto es una cuestión aún pendiente. 
Y ello, sin olvidar el imprescindible "conocimiento del contenido" sobre el complejo dominio de STEAM, además del conocimiento didáctico asociado, no ya del profesorado del ámbito científico-tecnológico, sino también de quienes nos dedicamos a la formación de este colectivo de profesorado (Toma y García-Carmona, 2019-en prensa). De acuerdo con lo señalado más arriba, los y las didactas tenemos una formación académica de origen muy dispar y específica en una disciplina concreta (biología, física, química, geología, matemáticas, ingeniería, ciencias ambientales, etc.). A esto hay que unir que, con más frecuencia de la deseable, en la formación de las y los futuros docentes del ámbito científico-tecnológico participa profesorado universitario que ni siquiera tiene la preparación y trayectoria académica necesarias en las didácticas correspondientes (Montero y García-Carmona, 2018).

Pues, incluso con todas estas limitaciones, me parece todavía más desconcertante que la educación enmarcada en STEAM se promueva desde la didáctica de la ciencia con una naturalidad y firmeza tales, que me atrevo a decir que se trata de un caso de "posverdad" dentro de una disciplina académica. Es lo que Richard Feynman denominó, hace más de 40 años, "ciencia del culto a la carga" (Feynman, 1974), en alusión a las "teorías" que, aun cuando no funcionan, se ponen de moda, se promueven y aceptan por una parte importante de la sociedad. Porque la cuestión clave es: ¿debe la didáctica de la ciencia promocionar un enfoque educativo que no ha superado aún unas pruebas mínimas de validación y consenso en nuestra comunidad educativa particular? $O$ en la misma línea de lo que Feynman (1974) argumenta para la investigación científica, en general, ¿por qué las propuestas educativas STEAM solo suelen informar de sus (posibles) idoneidades didácticas, y no de todos aquellos factores, de diversa índole, que pueden invalidar o mermar su eficacia educativa? Aunque, claro, para esto último es esencial que antes exista la suficiente investigación empírica en las aulas de nuestro contexto educativo; una circunstancia que, como he adelantado, no se da en estos momentos.

Es cierto que existen otros planteamientos o enfoques didácticos que se continúan promoviendo, aunque no han mostrado ser infalibles por motivos diversos. Estoy pensando, por ejemplo, en el ya mencionado enfoque educativo basado en la indagación (EBI). No obstante, a diferencia del enfoque STEAM, el EBI se presenta en la bibliografía con diversas conceptualizaciones que, aun con matices diferenciadores, todas muestran aspectos comunes bien fundamentados; los cuales, cuentan con un consenso generalizado, desde hace décadas, dentro de la comunidad internacional de didáctica de la ciencia (Ab-ElKhalick et al., 2004; Cañal, 2007; Garritz, 2012; Porlán, 2018; Rocard et al., 2007). Asimismo, la efectividad educativa del EBI ha sido ampliamente analizada de manera empírica en diversos contextos (Cañal, Travé y Pozuelos, 2011; Minner, Levy y Century, 2010; Rönnebeck, Bernholt y Ropohl, 2016; Zhang, 2016); de modo que son bien conocidas sus fortalezas, debilidades y, en consecuencia, las propuestas que serían necesarias para su mejora.

Si aún no estamos en este nivel de desarrollo investigativo con respecto al enfoque STEAM, ¿no sería conveniente ser más cautos en su proyección, y presentarlo con las mismas reservas que se tienen ante un "medicamento" que se halla en fase de ensayo clínico sobre su eficacia y seguridad para la salud? Como McComas y Burgin (2020) dicen, si el enfoque STEAM se está promoviendo sobre las débiles bases empíricas y epistemológicas que conocemos actualmente, se debería "reducir la velocidad" en su promoción.

\section{Complejos en la enseñanza de la ciencia ante STEAM}

Por otra parte, aunque muy ligado a todo lo tratado anteriormente, tengo la impresión de que ahora existe cierto complejo en el área de didáctica de la ciencia para hablar de "enseñanza de la ciencia" o "educación científica", como si fuera algo desfasado y con poca enjundia ante planteamientos más grandilocuentes y novedosos como "educación 
STEAM". Hasta hace muy poco, proponer a estudiantes que aborden una pregunta de investigación escolar sobre un fenómeno natural, que construyan y/o manejen algún sencillo artefacto para hacer mediciones, así como que se ayuden de herramientas matemáticas básicas y de ciertos recursos TIC, era asumido, sin ningún tipo de reservas, como algo propio de la enseñanza de la ciencia.

Cuando era profesor de Secundaria, llevé a cabo un pequeño proyecto escolar con mi alumnado de física y química, orientado por la siguiente pregunta: "¿Cómo podemos ver objetos detrás de obstáculos opacos?" (García-Carmona, 2011, cap. 5). El proyecto, que se desarrolló en un marco de aprendizaje por indagación, derivó en el diseño y la construcción de un doble periscopio "casero" (¿ingeniería escolar?), que precisó de conocimientos básicos de óptica, geometría y tecnología (técnicas, uso de herramientas, calibrado y funcionalidad, estética, etc.) recogidos en el currículo oficial de la ESO. Nunca me planteé que no estuviera haciendo algo enmarcado en ciencia escolar. A lo mejor, si llego a proponer ese proyecto ahora, igual lo hubiera etiquetado como "proyecto STEAM". Quién sabe. Si bien, para que eso sucediera, no tendría que contar con el "bagaje" didáctico para la enseñanza de la ciencia, acumulado durante años.

Sinceramente, no sé qué se gana desde un punto de vista pedagógico reetiquetando ahora como proyectos STEAM muchas de las propuestas que estaban enmarcadas en la enseñanza de la ciencia, ${ }^{10}$ salvo que adquieran validez muchas de las percepciones e impresiones expuestas más arriba. Lo cierto es que me sorprende, ya que la ciencia -y su transposición didáctica- se ha valido siempre de todos aquellos recursos, dispositivos y herramientas que ha tenido a su alcance para su desarrollo. Y no por ello se ha dejado de pensar que se estaba haciendo y aprendiendo ciencia. ¿Merece la pena, entonces, romper con asunciones que estaban bien consolidadas, en relación con lo que significa enseñar/ aprender ciencia?

Pienso también, al hilo de todo lo discutido, que la didáctica de la ciencia debería tomar una mayor concienciación y pregonar con el ejemplo: no podemos estar promoviendo entre el alumnado que la argumentación científica, las conclusiones de una investigación, etc. tienen que estar basadas en pruebas válidas y fiables, pero luego nuestros planteamientos didácticos sobre enseñanza de la ciencia desatienden esta máxima ineludible para cualquier disciplina científica. La consecuencia más directa es, en mi opinión, que se estará contribuyendo a eternizar, y agudizar aún más si cabe, la brecha entre investigación y práctica educativa que padecemos en la enseñanza de la ciencia. Porque, si las propuestas didácticas para su implementación en el aula no han sido previamente ensayadas y analizadas con rigor, es muy probable que luego no funcionen en las clases ordinarias, con la frustración y rechazo que ello puede conllevar entre el profesorado.

\section{Para terminar}

Quisiera aclarar que no he pretendido minusvalorar las buenas intenciones que posiblemente tengan muchos de quienes promueven el movimiento STEAM como un planteamiento educativo ideal. Tampoco he tratado de hacer una revisión sistemática de la bibliografía sobre el asunto porque ya existen en la literatura (algunos de ellas han sido citadas aquí; por ejemplo: Martín-Páez et al., 2019; Takeichi et al., 2020). El propósito de este trabajo ha sido, más bien, analizar con una mirada crítica particular la llegada del movimiento STEAM al ámbito educativo español, a partir de la revisión exhaustiva de una parte significativa de estudios y propuestas didácticas sobre este.

10 Para Carter (2017), "STEAM" se ha convertido en un neologismo de "educación científica". 
Por otra parte, soy consciente de que, en el debate sobre el futuro de la educación STEAM, como sucede con otros muchos asuntos educativos, hay quienes ven el vaso medio lleno, y quienes lo ven medio vacío. Personalmente, quisiera estar equivocado en mi percepción y tener razones suficientes para ser optimista, en tanto que el desarrollo e implementación del enfoque STEAM se traduzca en propuestas sólidas, bien fundamentadas y efectivas para mejorar la alfabetización científica de la ciudadanía. De hecho, a lo largo de mi trayectoria docente ha sido una constante desarrollar con mi alumnado planteamientos educativos basados en la integración de contenidos de diferentes áreas curriculares; principalmente enmarcados en el enfoque CTS (e.g., García-Carmona, 2005, 2006, 2008).

Sin embargo, mi escepticismo con el enfoque STEAM deriva de lo que proviene de la propia investigación educativa sobre este; y, especialmente, del panorama actual de la formación del profesorado de ciencia en España (Montero y García-Carmona, 2018). Hace años que se vienen apuntando las dificultades y mejoras necesarias en tal formación (Martín del Pozo y Porlán, 1999; Oliva y Acevedo, 2005); y de manera particular, con relación al profesorado de niveles educativos elementales. Incluso, hasta el punto de cuestionarse si la formación en didáctica de la ciencia es objetivamente viable con candidatas y candidatos a profesorado de Primaria (Barberà, 2002), a la vista de sus perfiles académicos habituales (Bonil y Márquez, 2011; Cañal et al., 2011; García-Carmona y Cruz-Guzmán, 2016; García-Carmona, Cruz-Guzmán y Criado, 2014; Verdugo, Solaz y Sanjosé, 2016).

Por tanto, me resulta inevitable pensar que el movimiento STEAM llega a la didáctica de la ciencia como una nueva "distracción" en el proceso de mejora que requiere, a todas luces, la educación científica básica en este país (MEFP, 2019). Soy plenamente consciente de todas las características que debería reunir un buen profesorado de ciencia (Gil y Vilches, 2004; Perales et al., 2014), y del camino que aún queda por recorrer para conseguirlas en nuestro contexto educativo (Banet, 2010; Cañal et al., 2013; COSCE, 2011; Montero y García-Carmona, 2018; Porlán, 2018). De modo que, cuando veo la impetuosa proclama en torno a la supuesta educación STEAM, se me viene a la mente el ya citado personaje de la comedia "El niño bien criado", y me pregunto: ¿De qué se pide los "huesos" al profesorado de ciencia, si aún no se ha hecho con la "carne"? Y lo que, quizá, sea más importante: ¿Se tiene claro cuál es esa "carne"? ¿Cómo se puede "cocinar" para que sea educativamente "digerible y nutritiva", en el aula?

Espero que este ensayo crítico contribuya a un debate profundo en torno a la promoción y posibilidades reales del enfoque STEAM, en el contexto educativo de este país. Lo necesitamos.

\section{Referencias}

Abd-El-Khalick, F., Boujaoude, S., Duschl, R., Lederman, N. G., Mamlok-Naaman, R., Hofstein, A., Niaz, M., Treagust, D, y Tuan, H.-L. (2004). Inquiry in science education: International perspectives. Science Education, 88(3), 397-419. DOI: https://doi. org/10.1002/sce.10118

Acevedo-Díaz, J. A. (1998). Análisis de algunos criterios para diferenciar entre ciencia y tecnología. Enseñanza de las Ciencias, 16(3), 409-420. Recuperado de: https:// www.raco.cat/index.php/Ensenanza/article/view/21546

Acevedo-Díaz, J. A. (2020). Todo lo que siempre quiso saber sobre STEM/STEAM y nunca se atrevió a preguntar. Formación IB. Recuperado de: http://formacionib. org/noticias/?Todo-lo-que-siempre-quiso-saber-sobre-STEM-STEAM-y-nunca-seatrevio-a-preguntar 
Acevedo-Díaz, J. A. y García-Carmona, A. (2016). Una controversia de la historia de la tecnología para aprender sobre naturaleza de la tecnología: Tesla vs. Edison-La guerra de las corrientes. Enseñanza de las Ciencias, 34(1), 193-209. DOI: http:// dx.doi.org/10.5565/rev/ensciencias.1916

Aikenhead, G. (2005). Educación Ciencia-Tecnología-Sociedad (CTS). Una buena idea como quiera que se le Ilame. Educación Química, 16(2), 304-315. DOI: http://dx.doi. org/10.22201/fq.18708404e.2005.2.66121

Aitken, J. y Mills, G. (1997). Tecnología creativa (trad. de Gillermo Solana). Madrid: Morata.

Akerson, V. L., Burgess, A., Gerber, A., Guo, M., Khan, T. A., y Newman, S. (2018). Disentangling the meaning of STEM: Implications for science education and science teacher education. Journal of Science Teacher Education, 29(1), 1-8. DOI: https://doi.org/1 0.1080/1046560X.2018.1435063

Banet, E. (2010). Finalidades de la educación científica en educación secundaria: aportaciones de la investigación educativa y opinión de los profesores. Enseñanza de las Ciencias, 28(2), 199-214. Recuperado de: https://www.raco.cat/index.php/ Ensenanza/article/view/199613

Barberá, O. (2002). El área de Didáctica de las Ciencias Experimentales: ¿Apuesta de futuro o error del pasado? Revista de Educación, 382, 97-109. Recuperado de: http:// www.educacionyfp.gob.es/revista-de-educacion/numeros-revista-educacion/ numeros-anteriores/2002/re328/re328-06.html

Bencze, L., Carter, L., Levinson, R., Martins, I., Pouliot, C., Weinstein, M. y Zouda, M. (2019). EcoJust STEM Education Mobilized Through Counter-Hegemonic Globalization. En K. J. Saltman y A. J. Means (eds.), The Wiley Handbook of Global Educational Reform (pp. 389-411). Hoboken, NJ: Wiley Backwell.

Bonil, J. y Márquez. C. (2011). ¿Qué experiencias manifiestan los futuros maestros sobre las clases de ciencias? Implicaciones para su formación. Revista de Educación, 354, 447-472. Recuperado de: http://10.0.17.86/1988-592X-RE-2011-354-007

Cañal, P. (2007). La investigación escolar, hoy. Alambique, 52, 9-19. Recuperado de: http:// hdl.handle.net/11162/22660

Cañal, P., Criado, A. M., García-Carmona, A. y Muñoz, G. (2013). La enseñanza relativa al medio en las aulas españolas de Educación Infantil y Primaria: Concepciones didácticas y práctica docente. Investigación en la Escuela, 81, 21-42. DOI: https:// doi.org/10.12795/IE.2013.i81.02

Cañal, P., Travé, G. y Pozuelos, F. J. (2011). Análisis de obstáculos y dificultades de profesores y estudiantes en la utilización de enfoques de investigación escolar. Investigación en la Escuela, 73, 5-26. DOI: https://doi.org/10.12795/IE.2011.i73.01

Carter, L. (2017). Neoliberalism and STEM education: Some Australian policy discourse. Canadian Journal of Science, Mathematics and Technology Education, 17(4), 247-257. DOI: https://doi.org/10.1080/14926156.2017.1380868

Cetto, A. M., Schneegans, S. y Moore, H. (eds.) (2000). World Conference on Science for the twenty-first century. A new commitment. Paris: UNESCO.

Chen, G. A. y Buell, J. Y. (2018). Of models and myths: Asian(Americans) in STEM and the neoliberal racial project. Race Ethnicity and Education, 21(5), 607-625. DOI: https:// doi.org/10.1080/13613324.2017.1377170 
Confederación de Sociedades Científicas de España [COSCE]. (2011). Informe sobre la enseñanza de las ciencias en la didáctica escolar para edades tempranas en España. Madrid: COSCE.

Criado, A. M. y García-Carmona, A. (2011). Investigando las máquinas y artefactos. Sevilla: Díada.

Czerniak, C. M., Weber, W. B., Sandmann, A., y Ahern, J. (1999). A literature review of science and mathematics integration. School Science and Mathematics, 99(8), 421-430. DOI: https://doi.org/10.1111/j.1949-8594.1999.tb17504.x

Dare, E. A., Ellis, J. A., y Roehrig, G. H. (2018). Understanding science teachers' implementations of integrated STEM curricular units through a phenomenological multiple case study. International Journal of STEM Education, 5(4), 1-19. DOI: https://doi. org/10.1186/s40594-018-0101-z

Dogan, B. y Robin, B. (2015). Technology's role in STEM education and the STEM SOS model. En A. Sahin (ed.), A Practice-based Model of STEM Teaching (pp. 77-94). Rotterdam: Sense.

Echeverría, J. (2005). La revolución tecnocientífica. CONfines, 1(2), 9-15. Recuperado de: http://www.scielo.org.mx/scielo.php?script=sci_arttext\&pid $=$ S1870-35692005000200001

Ejiwale, J. (2013). Barriers to successful implementation of STEM education. Journal of Education and Learning, 7(2), 63-74. DOI: http://dx.doi.org/10.11591/edulearn. v7i2.220

Feynman, R. (1974). Cargo cult science. Engineering and Science, 37(7), 10-13.

García-Carmona, A. (2001). Matemáticas: la musa de la física. Revista Española de Física, 15(2), 6-8.

García-Carmona, A. (2002). Ciencia y pensamiento ilustrado. Red Científica: Ciencia, Tecnología y Pensamiento. Recuperado de: https://www.researchgate.net/ publication/260185138_Ciencia_y_pensamiento_ilustrado

García Carmona, A. (2005). Relaciones CTS en el estudio de la contaminación atmosférica: una experiencia con estudiantes de secundaria. Revista Electrónica de Enseñanza de las Ciencias, 4(2), 1-17. Recuperado de: http://reec.uvigo.es/volumenes/ volumen4/ART3_Vol4_N2.pdf

García Carmona, A. (2006). Interacciones CTS en el aprendizaje del electromagnetismo: una experiencia para el desarrollo de actitudes de responsabilidad. Investigación en la Escuela, 58, 79-91. Recuperado de: https://revistascientificas.us.es/index.php/ IE/article/view/7320

García-Carmona, A. (2008). Relaciones CTS en la educación científica básica II. Investigando los problemas del mundo. Enseñanza de las Ciencias, 26(3), 389-402. Recuperado de: https://www.raco.cat/index.php/Ensenanza/article/view/132195

García-Carmona, A. (2011). Aprender física y química mediante secuencias de enseñanza investigadoras. Archidona (Málaga): Aljibe.

García-Carmona, A. (2020). Investigación basada en pruebas como antídoto de modas didácticas en la enseñanza de las ciencias. Góndola, Enseñanza y Aprendizaje de las Ciencias, 15(1), 5-7. DOI: https://doi.org/10.14483/23464712.15332 
García-Carmona, A. y Cruz-Guzmán, M. (2016). ¿Con qué vivencias, potencialidades y predisposiciones inician los futuros docentes de Educación Primaria su formación en la enseñanza de la ciencia? Revista Eureka sobre Enseñanza y Divulgación de las Ciencias, 13(2), 440-458. DOI: http://dx.doi.org/10.25267/Rev_Eureka_ensen_ divulg_cienc.2016.v13.i2.15

García-Carmona, A., Cruz-Guzmán, M. y Criado, A. M. (2014). “¿Qué hacías para aprobar los exámenes de ciencias, qué aprendiste y qué cambiarías?" Preguntamos a futuros docentes de educación primaria. Investigación en la Escuela, 84, 31-46. DOI: https://doi.org/10.12795/IE.2014.i84.03

Garratt, J. (1993). Diseño y tecnología. Madrid: Akal.

Garritz, A. (2012). Proyectos educativos recientes basados en la indagación de la química. Educación Química, 23(4), 458-464. DOI: http://dx.doi.org/10.22201/ fq.18708404e.2012.4.40460

Gil, D. (1994). El currículo de ciencias en la educación secundaria obligatoria: cárea o disciplinas? iNi lo uno ni lo otro, sino todo lo contrario! Infancia y Aprendizaje, 17(65), 19-30. DOI: https://doi.org/10.1174/02103709460575614

Gil, D. y Viches, A. (2004). La formación del profesorado de ciencias de secundaria y de universidad. La necesaria superación de algunos mitos bloqueadores. Educación Química, 15(1), 43-51. DOI: http://dx.doi.org/10.22201/ fq.18708404e.2004.1.66215

Guerra, J. M. (1984). Ciencia integrada en España: Un análisis del curriculum. Enseñanza de las Ciencias, 2(3), 170-174. Recuperado de: https://www.raco.cat/index.php/ Ensenanza/article/view/50755

Katona, E. (2017). La relatividad de los relojes. La (posible) influencia de Einstein sobre García Lorca, Dalí y Buñuel. En F. Dóra (ed.), Paralelismos e interpretaciones en torno a la Residencia de Estudiantes (pp. 69-91). Budapest: ELTE Eötvös Collegium.

Kelley, T. R., y Knowles, J. G. (2016). A conceptual framework for integrated STEM education. International Journal of STEM Education, 3(11), 1-11. DOI: https://doi.org/10.1186/ s40594-016-0046-z

Maiztegui, A., Acevedo, J. A., Caamaño, A., Cañal, P., Carvalho, A. P. M., del Carmen, L. et al. (2002). Papel de la tecnología en la educación científica: una dimensión olvidada. Revista Iberoamericana de Educación, 28, 129-155. DOI: https://doi.org/10.35362/ rie280962

Manchón-Gordón, A. F. y García-Carmona, A. (2018). ¿Qué investigación didáctica en el aula de física se publica en España? Una revisión crítica de la última década para el caso de educación secundaria. Enseñanza de las Ciencias, 36(2), 125-141. DOI: https://doi.org/10.5565/rev/ensciencias.2451

Margot, K. C. y Kettler, T. (2019). Teachers' perception of STEM integration and education: A systematic literature review. International Journal of STEM Education, 6(1), 1-16. DOI: https://doi.org/10.1186/s40594-018-0151-2

Martín del Pozo, R. y Porlán, R. (1999). Tendencias actuales en la formación inicial del profesorado sobre los contenidos escolares. Revista Interuniversitaria de Formación del Profesorado, 35, 115-128. Recuperado de: https://core.ac.uk/download/ pdf/51384247.pdf 
Martín-Gordillo, M. (2020). "Es necesario introducir la perspectiva ética y social en la formación STEAM del profesorado." (Entrevista). Austurias4STEAM. Recuperado de: https://asturias4steam.eu/marianomartingordillo_iesn5/

Martín-Páez, T., Aguilera, D., Perales-Palacios, F. J., y Vílchez-González, J. M. (2019). What are we talking about when we talk about STEM education? A review of literature. Science Education, 103(4), 799-822. DOI: https://doi.org/10.1002/sce.21522

McComas, W. F. y Burgin, S. R. (2020). A Critique of "STEM" Education. Science \& Education, 29(4), 805-829. DOI: https://doi.org/10.1007/s11191-020-00138-2

MEFP [Ministerio de Educación y Formación Profesional] (2019). Informe PISA 2018. Programa para la evaluación internacional de los estudiantes. Madrid: Secretaría General Técnica.

Minner, D. D., Levy, A. J., y Century, J. (2010). Inquiry-based science instruction -What is it and does it matter? Results from a research synthesis years 1984 to 2002. Journal of Research in Science Teaching, 47(4), 474-496. DOI: https://doi.org/10.1002/ tea.20347

Montero, L. y García-Carmona, A. (2018). Políticas, investigación y prácticas en la formación inicial del profesorado de ciencias en España. En A. Cachapuz, A. Shigunov e I. Fortunato (eds.) Formação inicial e continuada de professores de ciências: o que se pesquisa no Brasil, Portugal e Espanha. São Paulo (Brasil): Edições Hipótese.

Morales-Doyle, D. y Gutstein, E. R. (2019). Racial capitalism and STEM education in Chicago Public Schools. Race Ethnicity and Education, 22(4), 525-544. DOI: https://doi.org/ 10.1080/13613324.2019.1592840

Ocaña, G., Romero, I. M., Gil, F. y Codina, A. (2015). Implantación de la nueva asignatura "Robótica" en Enseñanza Secundaria y Bachillerato. Investigación en la Escuela, 87, 65-79. DOI: https://doi.org/10.12795/IE.2015.i87.05

Oliva, J. M., y Acevedo, J. A. (2005). La enseñanza de las ciencias en primaria y secundaria hoy. Algunas propuestas de futuro. Revista Eureka sobre Enseñanza y Divulgación de las Ciencias, 2(2), 241-250. DOI: http://dx.doi.org/10.25267/Rev_Eureka_ensen_ divulg_cienc.2005.v2.i2.10

Organization for Economic Co-operation and Development [OECD]. (2006). Assessing scientific, reading and mathematical literacy: A framework for PISA 2006. Paris: OECD Publishing.

Organization for Economic Co-operation and Development [OECD]. (2019). PISA 2018 assessment and analytical framework. Paris: OECD Publishing.

Ortiz-Revilla, J., Adúriz-Bravo, A. y Greca, I.M. (2020). A framework for epistemological discussion on integrated STEM education. Science \& Education, 29(4), 857-880. DOI: https://doi.org/10.1007/s11191-020-00131-9

Pastor, I. (2018). Metodología STEM a través de la percepción docente (Trabajo Fin de Máster). Universidad de Valladolid, Valladolid.

Perales, F. J. y Aguilera, D. (2020). Ciencia-Tecnología-Sociedad vs. STEM: ¿evolución, revolución o disyunción? Ápice. Revista de Educación Científica, 4(1), 1-15. DOI: https://doi.org/10.17979/arec.2020.4.1.5826

Perales, F. J., Cabo, J. M., Vílchez, J. M., Fernández-González, M., González-García, F. y Jiménez-Tejada, P. (2014). La reforma de la formación inicial del profesorado de 
ciencias: Propuesta de un diseño del currículo basado en competencias. Enseñanza de las Ciencias, 32(1), 9-28. DOI: http://dx.doi.org/10.5565/rev/ensciencias.898

Pleasants, J. (2020). Inquiring into the nature of STEM problems. Science \& Education, 29(4), 831-855. DOI: https://doi.org/10.1007/s11191-020-00135-5

Pleasants, J., Clough, M. P., Olson, J. K. y Miller, G. (2019). Fundamental issues regarding the nature of technology. Implications for STEM education. Science \& Education, 28(35), 561-597. DOI: https://doi.org/10.1007/s11191-019-00056-y

Porlán, R. (2018). Didáctica de las ciencias con conciencia. Enseñanza de las Ciencias, 36(3), 5-22. DOI: https://doi.org/10.5565/rev/ensciencias.2795

Radloff, J. y Guzey, S. (2016). Investigating preservice STEM teacher conceptions of STEM education. Journal of Science Education and Technology, 25(5), 759-774. DOI: https://doi.org/10.1007/s10956-016-9633-5

Raynaud, D. (2018). ¿Qué es la tecnología? Pamplona: Laetoli.

Reynante, B. M., Selbach-Allen, M. E. y Pimentel, D. R. (2020). Exploring the promises and perils of integrated STEM through disciplinary practices and epistemologies. Science \& Education, 29(4), 785-803. DOI: https://doi.org/10.1007/s11191-020-00121-x

Rocard, M., Csermely, P., Jorde, D., Lenzen, D., Walberg, H. y Hemmo, V. (2007). Science education now: A renewed pedagogy for the future of Europe. Brussels: Directorate General for Research, Science, Economy and Society.

Rönnebeck, S., Bernholt, S., y Ropohl, M. (2016). Searching for a common ground-A literature review of empirical research on scientific inquiry activities. Studies in Science Education, 52(2), 161-197. DOI: https://doi.org/10.1080/03057267.2016. 1206351

Rossi, A. y Barajas, M. (2015). Elección de estudios CTIM y desequilibrios de género. Enseñanza de las Ciencias, 33(3), 59-76. DOI: http://dx.doi.org/10.5565/rev/ ensciencias.1481

Sánchez Ron, J. M. (2016). Belleza y verdad. Minerva, 26, 80-90. Recuperado de: https:// www.circulobellasartes.com/mediateca/belleza-y-verdad/

Sanders, M. (2009). STEM, STEM education, STEM mania. Technology Teacher, 68(4), 20-26. Recuperado de: https://vtechworks.lib.vt.edu/bitstream/handle/10919/51616/ STEMmania.pdf?sequence

Sharma, A. (2016). STEM-ification of Education: The Zombie Reform Strikes Again. Journal for Activist Science \& Technology Education, 7(1), 42-51. Recuperado de: https:// jps.library.utoronto.ca/index.php/jaste/article/view/26826

Stevenson, H. J. (2015). STEM education ins not only an integrated curriculum: STEM as part of an integrated neoliberal agenda. En M. Abendroth y B. L. Porfilio (eds.), Understanding Neoliberal Rule in K12 Schools: Educational Fronts for Local and Global Justice (pp. 179-196). Charlotte, NC: IAP.

Takeuchi, M. A., Sengupta, P., Shanahan, M.-C., Adams, J. D. y Hachem, M. (2020). Transdisciplinarity in STEM education: a critical review. Studies in Science Education, 56(2), 213-253. DOI: https://doi.org/10.1080/03057267.2020.1755802

Toma, R. B. y García-Carmona, A. (2019-en prensa). "De STEM nos gusta todo, menos STEM". Análisis crítico de una tendencia educativa de moda. Enseñanza de las Ciencias. 
Toma, R. B. y Greca, I. M. (2018). The effect of integrative STEM instruction on elementary students' attitudes toward science. Eurasia Journal of Mathematics, Science and Technology Education, 14(4), 1383-1395. DOI: https://doi.org/10.29333/ ejmste/83676

Vakil, S., y Ayers, R. (2019). The racial politics of STEM education in the USA: interrogations and explorations. Race Ethnicity and Education, 22(4), 449-458. DOI: https://doi. org/10.1080/13613324.2019.1592831

Valdeolmillos, C. (2019, 12 abril). La ciencia española, presente en el equipo que ha fotografiado un agujero negro. MCPRO. Recuperado de: https://www. muycomputerpro.com/2019/04/12/la-ciencia-espanola-presente-en-el-equipoque-ha-fotografiado-un-agujero-negro

Vázquez-Alonso, A., Acevedo-Díaz, J. A. y Manassero-Mas, M. A. (2005). Más allá de la enseñanza de las ciencias para científicos: hacia una educación científica humanística. Revista Electrónica de Enseñanza de las Ciencias, 4(2), 1-30. Recuperado de: http:// reec.uvigo.es/volumenes/volumen4/ART5_Vol4_N2.pdf

Vázquez, A. y Manassero Mas, M. A. (2015). La elección de estudios superiores científicotécnicos: análisis de algunos factores determinantes en seis países. Revista Eureka sobre Enseñanza y Divulgación de las Ciencias, 12(2), 264-277. DOI: http://dx.doi. org/10.25267/Rev_Eureka_ensen_divulg_cienc.2015.v12.i2.03

Verdugo, J. J., Solaz, J. J. y Sanjosé, V. (2016). Pre-service primary school teachers' science content knowledge: An instrument for its assessment. International Journal of Innovation in Science and Mathematics Education, 24(2), 37-51. Recuperado de: http://hdl.handle.net/10550/55057

Weinberg, S. (2010). El sueño de una teoría final. Barcelona: Crítica.

Wilson, E. O. (1999). Consilence. La unidad del conocimiento. Barcelona: Círculo de Lectores.

Zhang, L. (2016). Is inquiry-based science teaching worth the effort? Some thoughts worth considering. Science \& Education, 25(7-8), 897-915. DOI: https://doi.org/10.1007/ s11191-016-9856-0 